\title{
Adipokines, Biomarkers of Endothelial Activation, and Metabolic Syndrome in Patients with Ankylosing Spondylitis
}

\author{
Fernanda Genre, ${ }^{1}$ Raquel López-Mejías, ${ }^{1}$ José A. Miranda-Filloy, ${ }^{2}$ Begoña Ubilla, ${ }^{1}$ \\ Beatriz Carnero-López, ${ }^{3}$ Ricardo Blanco, ${ }^{1}$ Trinitario Pina, ${ }^{1}$ Carlos González-Juanatey, ${ }^{4}$ \\ Javier Llorca, ${ }^{5}$ and Miguel A. González-Gay ${ }^{1}$
}

${ }^{1}$ Epidemiology, Genetics and Atherosclerosis Research Group on Systemic Inflammatory Diseases, Rheumatology Division, IDIVAL, 39011 Santander, Spain

${ }^{2}$ Rheumatology Division, Hospital Lucus Augusti, 27003 Lugo, Spain

${ }^{3}$ Oncology Division, Hospital del Bierzo, 24411 Ponferrada, León, Spain

${ }^{4}$ Cardiology Division, Hospital Lucus Augusti, 27003 Lugo, Spain

${ }^{5}$ Computational Biology, School of Medicine, University of Cantabria, IDIVAL, and CIBER Epidemiología y Salud Pública (CIBERESP), 39011 Santander, Spain

Correspondence should be addressed to Miguel A. González-Gay; miguelaggay@hotmail.com

Received 7 January 2014; Accepted 13 February 2014; Published 18 March 2014

Academic Editor: Lorenzo Cavagna

Copyright (C) 2014 Fernanda Genre et al. This is an open access article distributed under the Creative Commons Attribution License, which permits unrestricted use, distribution, and reproduction in any medium, provided the original work is properly cited.

Ankylosing spondylitis (AS) is a chronic inflammatory rheumatic disease associated with accelerated atherosclerosis and increased risk of cardiovascular (CV) disease. AS patients also display a high prevalence of features clustered under the name of metabolic syndrome (MeS). Anti-TNF- $\alpha$ therapy was found to be effective to treat AS patients by suppressing inflammation and also improving endothelial function. Previously, it was demonstrated that a short infusion of anti-TNF- $\alpha$ monoclonal antibodyinfliximab induced a rapid and dramatic reduction in serum insulin levels and insulin resistance along with a rapid improvement of insulin sensitivity in nondiabetic AS patients. The role of adipokines, MeS-related biomarkers and biomarkers of endothelial cell activation and inflammation seem to be relevant in different chronic inflammatory diseases. However, its implication in AS has not been fully established. Therefore, in this review we summarize the recent advances in the study of the involvement of these molecules in CV disease or MeS in AS. The assessment of adipokines and biomarkers of endothelial cell activation and MeS may be of potential relevance in the stratification of the CV risk of patients with AS.

\section{Introduction}

Ankylosing spondylitis (AS) is a chronic inflammatory rheumatic disease, which mainly affects the axial joints, including the spine, sacroiliac joints, and entheses, but it may also involve peripheral joints [1]. Along with disease progression, inflamed joints tend to fuse (ankylosis) and there is also an ossification of the inflamed entheses, often leading to a loss of the well-known flexibility of the spine. AS is more prevalent in men than in women and usually appears around the third decade of life [1]. Moreover, extra-articular manifestations such as uveitis, psoriasis, or osteoporosis are frequently associated with this rheumatologic disease [2].
As observed in other rheumatologic diseases, such as rheumatoid arthritis (RA), AS patients disclose an increased risk of cardiovascular (CV) disease when compared to general population, being $\mathrm{CV}$ diseases one of the main causes of mortality in these patients [1]. Furthermore, an accelerated atherosclerotic process in these patients has also been reported [3].

AS patients also display a high prevalence of features such as obesity, dyslipidemia, hypertension, alterations in glucose metabolism, and insulin resistance (IR), which are clustered under the name of metabolic syndrome (MeS) [4]. Interestingly, individuals that suffer MeS also exhibit a dysregulation of adipokines, which are highly bioactive 
substances secreted by adipocytes and immune cells and that are involved not only in metabolic functions but that also play an immunomodulatory role $[5,6]$. This dysregulation leads to metabolic disorders such as IR [5], an essential feature of MeS that has been associated with inflammation [7]. In addition, multiple evidences show that IR promotes endothelial dysfunction $[8,9]$, an early key step in the atherogenic process which appears even before the structural changes associated with this process [10].

Regarding therapeutic approaches aimed to treat AS, antiTNF- $\alpha$ therapy was found to be effective to treat patients with this disease and other types of spondyloarthritis [11-13]. AntiTNF- $\alpha$ agents neutralize this cytokine leading to suppression of inflammation and, consequently, to a reduction of disease activity [14]. Moreover, it was demonstrated that this biologic therapy improves endothelial function in AS patients [15].

For the purpose of this review, we took advantage of data obtained from a series of 30 nondiabetic AS patients undergoing anti-TNF- $\alpha$ therapy with the chimeric anti-TNF$\alpha$ monoclonal antibody infliximab [16]. At the time of assessment, these patients had been treated with this biologic agent for a median of 23 months. Since IR promotes endothelial dysfunction $[8,9]$, while anti-TNF- $\alpha$ treatment improves endothelial function in AS patients [15], our first objective was to evaluate short-term insulin response following antiTNF- $\alpha$ infliximab therapy. We observed that our patients experienced a rapid and dramatic reduction in serum insulin levels and IR along with rapid improvement of insulin sensitivity after a single administration of infliximab [16]. This observation had previously been described in patients with RA undergoing anti-TNF- $\alpha$ infliximab therapy $[17,18]$.

Considering these results, we decided to further evaluate the short-term effect of anti-TNF- $\alpha$ therapy in our series of AS patients on periodical treatment with infliximab on $\mathrm{MeS}$ related biomarkers, adipokines, and biomarkers of endothelial cell activation and inflammation. Figure 1 depicts the pathophysiologic context that encompasses all the molecules reviewed in this paper. Furthermore, the main results derived from these studies on the effect of an infliximab infusion are summarized in Table 1.

In this review, recent advances in the study of the involvement of these molecules in CV disease or MeS in AS patients are discussed, along with the possible link between these biomarkers and/or adipokines and clinical characteristics of this rheumatic disease.

\section{Metabolic Syndrome-Related Biomarkers in AS}

As previously mentioned, AS patients frequently display features of MeS [4]. Therefore, the study of potential biomarkers involved in the development of such features and its association with the pathogenesis of this spondyloarthritis could give us hints for the outcome and treatment of these patients.

2.1. Ghrelin. Ghrelin, a peptide predominantly expressed in the stomach, is the endogenous ligand for the growth
TABLE 1: Effect of an infusion of the anti-TNF- $\alpha$ monoclonal antibody infliximab on MeS-related biomarkers, biomarkers of endothelial cell activation and inflammation, and adipokines in a series of AS patients undergoing periodic treatment with this drug.

\begin{tabular}{ll}
\hline Target & Biologic effect \\
\hline $\begin{array}{l}\text { MeS-related } \\
\text { biomarkers }\end{array}$ & $\begin{array}{l}\text { Reduction in serum insulin levels and IR } \\
\text { Improvement of insulin sensitivity } \\
\text { Reduction of RBP-4 serum levels } \\
\text { No significant change on ghrelin serum } \\
\text { levels }\end{array}$ \\
\hline $\begin{array}{l}\text { Biomarkers of } \\
\text { endothelial } \\
\text { cell activation and } \\
\text { inflammation }\end{array}$ & $\begin{array}{l}\text { Reduction of Angpt-2 serum levels } \\
\text { No significant change on ADMA and GSN } \\
\text { serum levels } \\
\text { No significant reduction on OPG plasma } \\
\text { levels }\end{array}$ \\
\hline & $\begin{array}{l}\text { No significant change in the levels of the } \\
\text { different adipokines (adiponectin, resistin, } \\
\text { leptin, visfatin, and apelin) }\end{array}$ \\
\hline
\end{tabular}

hormone secretagogue receptor (GHS-R), which regulates food intake and $\mathrm{GH}$ expression [19] and also acts as an anti-inflammatory molecule [20]. Previously, low levels of this peptide have been observed in obese individuals [21], a condition directly associated with hyperinsulinemia and IR. In our series we disclosed a significant correlation between ghrelin and IR and insulin sensitivity [22]. Similar results had previously been reported in individuals without rheumatic diseases [23,24]. However, since only $10 \%$ of our patients were obese, possibly other mechanisms different from obesity may account for our findings. Furthermore, in this study we also observed a positive correlation between ghrelin and resistin, which is in accordance with their role in glucose homeostasis and the inflammatory process [22].

To our knowledge, the only previous study performed to evaluate ghrelin levels in AS patients was the one reported by Toussirot et al. [25]. However, in that study they described higher levels of ghrelin in AS patients when compared to controls. These results seem to be unexpected, since ghrelin is known to inhibit the production of inflammatory cytokines [20]. In line with this, RA patients show decreased ghrelin levels when compared to healthy controls [26]. Therefore, further studies are needed to elucidate whether the different inflammatory burden in RA and AS may account for these contradictory results.

We previously reported a significant elevation of ghrelin serum concentration upon a single infliximab infusion in RA patients with severe disease who despite receiving this antiTNF- $\alpha$ agent had active disease with persistent elevation of laboratory markers of inflammation [27]. However, following a single infusion of infliximab, when we compared ghrelin levels found immediately before and after an infliximab infusion (the drug was administered in the fasting state, in saline solution over 120 minutes), we only disclosed a mild but not significant increase of ghrelin serum concentration in patients with AS [22]. These disparate results may be due to the absence of severe disease in our series of patients of AS 


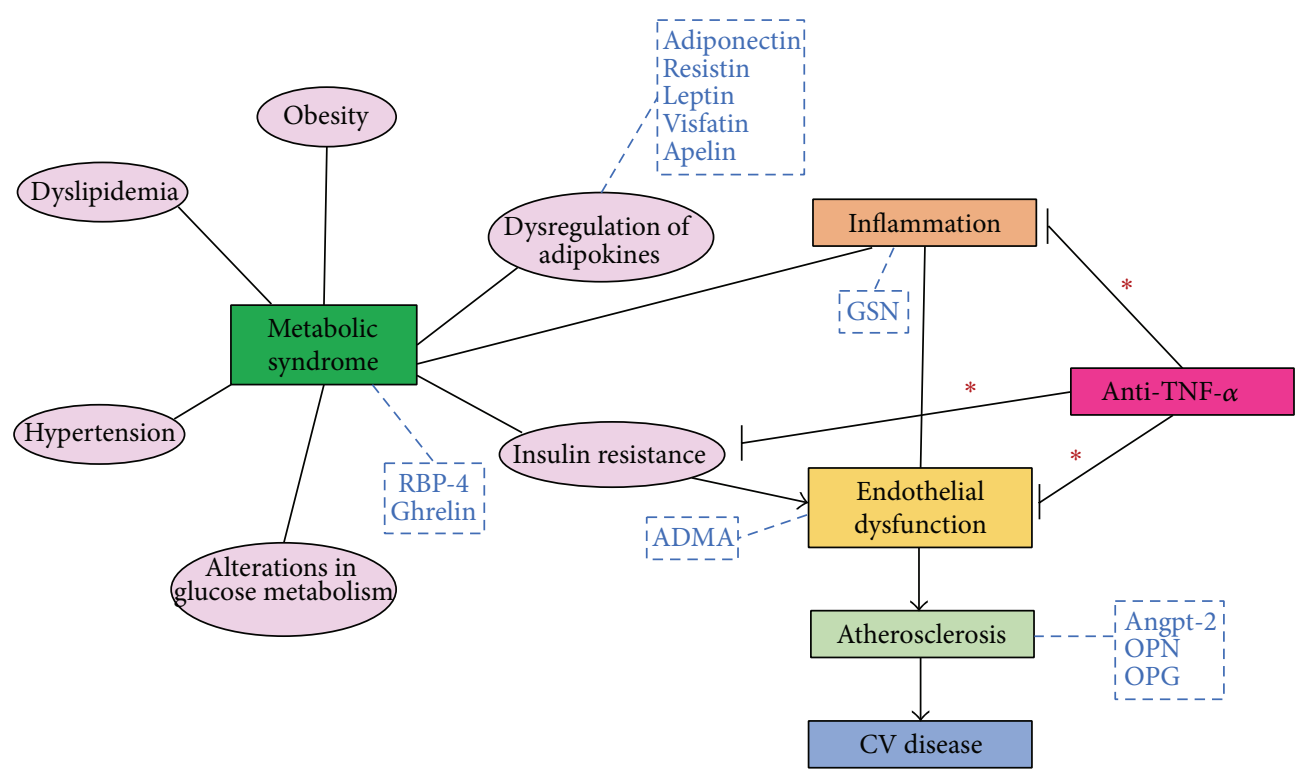

FIGURE 1: Pathophysiologic context that encompasses all the molecules reviewed in this paper. Ankylosing spondylitis patients display a high incidence of features clustered under the name of metabolic syndrome, which include obesity, dyslipidemia, hypertension, alterations in glucose metabolism, including insulin resistance, and also a dysregulation of adipokines. Moreover, all these pathologic features are associated with inflammation and lead to endothelial dysfunction and, consequently, to an enhanced risk of CV disease (mainly due to accelerated atherosclerosis) and CV death in these patients. Anti-TNF- $\alpha$ treatment not only suppresses inflammation, reducing thus ankylosing spondylitis activity, but it also improves endothelial function in these patients. The molecules that will be reviewed in this paper are included in this figure inside blue dashed boxes. ${ }^{*}$ Anti-TNF- $\alpha$ improves insulin resistance and endothelial function and also reduces inflammation. ADMA: asymmetric dimethylarginine; Angpt-2: angiopoietin-2; OPG: osteoprotegerin; OPN: osteopontin; RBP-4: retinol binding protein-4.

at the time of assessment. It was not the case in RA patients undergoing infliximab therapy as the series of patients with RA still had active disease despite periodical treatment with this TNF- $\alpha$ inhibitor.

\subsection{Retinol Binding Protein-4 (RBP-4). Retinol binding} protein-4 (RBP-4) is another metabolic syndrome-related biomarker, also considered a new potential cardiometabolic risk factor. This proinflammatory protein is mainly released by adipocytes but also expressed in liver and macrophages [28] and has been associated with IR in individuals with obesity, impaired glucose tolerance or type 2 diabetes mellitus, and nonobese subjects with or without family history of type 2 diabetes [29-31]. In our study, we disclosed a marginally significant correlation between RBP-4 serum levels and IR and a correlation with systolic blood pressure. Besides, when our AS patients where stratified according to sex, men showed higher levels of this protein than women [32], which was in keeping with the results previously reported by Gavi et al. [30]. More importantly, when we evaluated the effect of a single infusion of anti-TNF- $\alpha$ infliximab on RBP- 4 levels, we observed a statistically significant reduction in its levels. Furthermore, this change was more evident in those patients who had a higher IR index [32].

A former study disclosed that AS patients may have lower RBP-4 serum levels than controls [33]. However, when we compared our patients with healthy controls we did not find significant differences in RBP-4 serum levels [32]. The low disease activity at the time of study in our series of AS patients may explain the absence of differences in RBP-4 serum levels when compared with controls.

\section{Adipokines in AS}

Immune system and metabolism are linked through a network of soluble mediators widely known as adipokines, which take part in both metabolic and immunomodulatory functions [6]. Although the role of adipokines seems to be relevant in many chronic inflammatory diseases, the implication in AS has not been completely elucidated.

3.1. Adiponectin. Adiponectin is an adipokine mainly produced by adipocytes but that can also be found in endothelial cells, skeletal muscle cells, and cardiac myocytes [6]. Circulating adiponectin levels inversely correlate with adiposity [5], which suggests that this adipokine exerts a protective function against $\mathrm{CV}$ disease and obesity. Adiponectin increases fatty acid oxidation and reduces the synthesis of glucose in the liver and other tissues [34]. Depending on the context, this adipokine can have pro- or anti-inflammatory functions. Unlike observations in nonrheumatic patients, RA high levels of this adipokine have been reported in the inflamed joints, since it promotes matrix degradation [35]. However, results obtained in AS are contradictory. Some groups did not find differences in serum adiponectin levels between AS patients with active disease and controls [25], while others found 
significantly higher levels of this adipokine in a series of AS patients under treatment with infliximab, when compared with controls [36].

In our series of AS patients we found a positive correlation between adiponectin serum levels and insulin sensitivity, suggesting that low circulating adiponectin concentrations may be associated with metabolic abnormalities that promote CV disease in AS [37]. The effect of adiponectin on insulin sensitivity is mediated in part by its ability to activate signaling pathways that lead to glucose uptake in muscle tissue and the inhibition of gluconeogenesis in the liver [38]. Furthermore, AS patients with hip involvement or synovitis and/or enthesitis in other peripheral joints had higher levels of adiponectin than those who did not have these complications [37]. These results are in keeping with the proinflammatory role described for adiponectin in the joints of RA patients [35]. Therefore, higher adiponectin levels might help to establish a subgroup of AS patients with predominant peripheral involvement.

As observed in RA patients undergoing anti-TNF- $\alpha$ infliximab therapy [39], a single infusion of this biologic agent did not lead to significant changes in the serum levels of adiponectin in patients with AS [37].

\subsection{Resistin. Resistin is another proinflammatory adipokine} mainly produced by monocytes and macrophages [40]. Proinflammatory factors such as TNF- $\alpha$ and IL- 6 induce its expression [41]. Interestingly, a positive correlation between serum resistin and C-reactive protein (CRP) and erythrocyte sedimentation rate (ESR) has been observed in RA patients [42-44]. Moreover, resistin serum levels have been found increased in synovial fluid of RA patients [45]. In AS patients, likewise, higher levels of this adipokine have been observed as compared to controls [46].

In our cohort of AS patients treated with the TNF- $\alpha$ antagonist infliximab we did not observe any correlation between resistin concentration and disease activity or laboratory markers of inflammation, probably due to the low inflammatory burden as a result of prolonged treatment with anti-TNF- $\alpha$ [37]. These results are in agreement with those obtained by Kocabas et al., who did not find any correlation between resistin concentration and ESR, CRP, or BASDAI in their series of AS patients [46]. Once again, the lower inflammatory burden in our AS patients due to prolonged treatment with this biologic therapy could also explain the lack of effect observed after a single infusion of infliximab on resistin concentration [37].

3.3. Leptin. Leptin also belongs to the group of proinflammatory adipokines, being produced by many cell types, including adipocytes [47]. This adipokine is involved in body weight regulation, since it inhibits food intake and stimulates energy expenditure [48]. Moreover, a proatherogenic role for leptin has been described [49]. Leptin induces the production of the proinflammatory cytokines IL- 6 , IL-12, and TNF- $\alpha$ by monocytes and macrophages [50], while it suppresses the production of IL-4, an anti-inflammatory cytokines [51]. Leptin levels are regulated by inflammatory mediators such as TNF- $\alpha$ and IL-1 [52]. While in RA leptin has a clear proinflammatory role, showing increased levels in patients when compared with healthy controls [53], the results obtained for this adipokine in AS are contradictory. Some groups found increased leptin levels in AS patients with active disease when compared to controls [54], while others found lower circulating levels of this adipokine than in controls $[25,55]$.

In contrast to previous reports which found a correlation between leptin levels and BASDAI in a series of AS patients [54], in our AS cohort, we did not observe any correlation between the levels of this adipokine and clinical and laboratory parameters of disease activity and inflammation [56]. Similar results were obtained in a series of RA patients undergoing periodical anti-TNF- $\alpha$ therapy [57]. Furthermore, as previously described [58], when our series of AS patients were stratified according to sex, we disclosed higher levels of leptin in women [56].

When we analyzed the effect of TNF- $\alpha$ blockade on circulating leptin levels, we found that they were not significantly altered [56]. Similar results were obtained by other groups, either in AS patients after 6 months of infliximab treatment [36] or in RA patients undergoing 2 weeks and 6 months TNF- $\alpha$ blockade [59].

3.4. Visfatin. Visfatin, also known as pre-B cell colonyenhancing factor or PBEF, is a proinflammatory adipokine with ubiquitous expression [60]. Visfatin was reported to act as an insulin-mimetic adipokine [61]. This adipokine positively correlates with visceral fat [62] and has also been described as an immunomodulatory molecule [63, 64]. In fact, visfatin can induce monocytes to produce proinflammatory cytokines such as IL-1, TNF, and IL-6 [64]. Previously, increased levels of this adipokine have been observed in patients with RA [53] when compared to controls. However, to our knowledge, in addition to our study [56], the only previous study performed in AS patients was performed by Hulejová et al. [65]. In that study, which included AS patients with mild to moderate disease, no correlation was found between visfatin levels and disease activity, functional status, or acute-phase reactants [65]. Similarly, in our series of AS patients on periodical treatment with the anti-TNF$\alpha$-blocker infliximab, we could not find correlation between serum visfatin levels and clinical and laboratory parameters of disease activity and inflammation [56]. This was also the case for RA patients undergoing anti-TNF- $\alpha$ infliximab therapy [66].

Although we could not find association between visfatin serum levels and metabolic syndrome in RA patients with severe disease undergoing anti-TNF- $\alpha$ therapy [66], we observed a positive correlation between visfatin serum levels and IR in AS [56]. A former study performed in lean women with polycystic ovary syndrome obtained similar results [67]. However, in our series of AS patients visfatin levels did not change upon infliximab administration [56].

3.5. Apelin. Apelin is a quite recently new adipokine produced by diverse cell types, including adipocytes and endothelial cells [68]. This adipokine is considered a potential 
biomarker for $\mathrm{CV}$ disease risk since it stimulates nitric oxide (NO) release and, therefore, triggers arterial vasodilation [69]. Moreover, insulin directly upregulates the expression of apelin [70], making this adipokine an attractive candidate to be studied in metabolic disorders such as type-2 diabetes. Furthermore, low apelin levels have been associated with high LDL levels [71] and biomarkers of endothelial cell activation such as VCAM-1 and E-selectin correlated to apelin levels [72].

It has been postulated that apelin may have a role in the pathogenesis of the $\mathrm{CV}$ disease, since low levels of this adipokine have been observed in patients with ischemic heart disease [73]. However, contradictory results have been reported in patients with type 2 diabetes mellitus. In this regard, in a study fasting plasma apelin levels correlated positively with IR in patients with type 2 diabetes mellitus [74], while in another study plasma apelin levels were reduced in newly diagnosed and untreated patients with type 2 diabetes mellitus [75].

di Franco et al. measured apelin levels in early stage RA patients and found that they were lower than those observed in controls [76], suggesting a potential involvement of apelin in the pathogenesis of the rheumatic diseases. However, regarding AS, there are no previous reports performed to evaluate the levels of this adipokine in this disease. In a study of our group, apelin levels showed no association with markers of disease activity or MeS in patients with AS [77]. In line with our results, Ferraz-Amaro et al. did not find any correlation between the levels of apelin in RA patients treated with anti-TNF- $\alpha$ and BMI or IR [78].

In a further step, we analyzed the potential effect of anti-TNF- $\alpha$ infliximab treatment on apelin concentration in our series of AS patients. We found that even after the administration of a single dose of infliximab apelin serum levels were reduced, this decrease did not achieve statistical significance [77]. Similar results were previously described in RA patients after 12 months of anti-TNF- $\alpha$ treatment [78] or after 12 months of treatment with disease modifying antirheumatic drugs [76].

\section{Biomarkers of Endothelial Cell Activation and Inflammation}

In physiological conditions, $\mathrm{NO}$ acts as an anti-inflammatory molecule, maintaining the vascular wall in a quiescent state, also avoiding cellular proliferation. However, in the presence of inflammation or CV risk factors such as hypertension, hypercholesterolemia, or diabetes, the quiescent endothelium can switch to an activated phenotype. This leads to the secretion of proinflammatory factors such as cytokines and adipokines, to the expression of adhesion molecules for the recruitment of inflammatory cells to the vascular wall and to the generation of reactive oxygen species [79]. This pathological inflammatory condition also leads to a reduction in the release of $\mathrm{NO}$ into the arterial wall, either affecting its synthesis or due to oxidative inactivation of NO [80, 81], which further enhances the inflammatory status and maintains the endothelial activated phenotype [79].

Many biomarkers of endothelial cell activation and inflammation may be potentially used by clinicians to make an early diagnosis of $\mathrm{CV}$ disease and MeS. The implication of these biomarkers in the pathogenesis of the rheumatic diseases is also an issue of potential interest. However, as previously mentioned for adipokines, studies performed on AS patients are limited.

4.1. Asymmetric Dimethylarginine (ADMA). Asymmetric dimethylarginine (ADMA) is an endogenous inhibitor of the nitric oxide synthase (NOS), causing therefore a reduction in NO production and leading to endothelial dysfunction and $\mathrm{CV}$ events [82]. Consequently, ADMA has been proposed as a biomarker for endothelial dysfunction and a risk factor for CV disease $[82,83]$. Furthermore, increased levels of ADMA have been associated with hypertension [84], hypertriglyceridemia [85], hypercholesterolemia [86], diabetes mellitus [87], and IR [88], as well as with inflammatory diseases such as RA [76] and AS [89-91].

We found higher concentrations of ADMA in AS patients with hypertension [92]. Unexpectedly, we observed a significant negative correlation between ADMA levels and total cholesterol (TC) and LDL-cholesterol in AS [92], which apparently seems to contradict previous results that associated high ADMA levels with patients with hypercholesterolemia [86]. We think that this negative association between ADMA and TC and LDL-cholesterol might probably be the result of the long-term treatment with anti-TNF- $\alpha$ in our series of AS patients (almost 2 years), which may have led to a reduction of the inflammatory burden in these patients [14] along with complex lipidic changes [93, 94]. With respect to this, our series of patients with AS were in a state of low disease activity, with low levels of CRP, showing a BASDAI less than 3, which is indicative of a favorable disease activity state [92]. This could be the reason why we could not see statistically significant associations between CRP/disease activity markers and ADMA levels in our series of patients.

Furthermore, we did not observe any change on ADMA levels after a single infusion of anti-TNF- $\alpha$ [92]. This was in accordance with previous studies that described no effect on ADMA concentration following long-term TNF- $\alpha$ blockade $[95,96]$.

4.2. Angiopoietin-2 (Angpt-2). Angiopoietin-2 (Angpt-2) is a proinflammatory marker of endothelial cell activation that is involved in angiogenesis and makes the endothelium responsive to inflammatory cytokines [97]. Angpt-2 levels have been found increased in recent onset RA patients with CV disease when compared with those without CV disease [97], which suggests that it could be a potential biomarker for the development of CV disease. Interestingly, a recent study of our group disclosed a correlation between age at the time of disease onset in patients with RA and Angpt-2 levels [98]. More importantly, after adjustment for sex, age at RA diagnosis,and CV risk factors, Angpt-2 levels were higher in RA patients with CV disease than in RA patients 
without CV complications [98]. Interestingly, in our series of AS patients undergoing infliximab therapy, we also found an association between Angpt-2 serum levels and the age at the onset of symptoms of AS, as well as a marginally significant association with disease duration [99].

It has been reported that Angpt-2 promotes the proinflammatory activation of human macrophages in RA synovial tissue and that the neutralization of this molecule in an in vivo model of RA decreased disease severity, inflammation, neovascularisation, and joint destruction [100]. In line with this observation, we disclosed that a single infusion of antiTNF- $\alpha$ infliximab was associated with a dramatic reduction of Angpt-2 serum levels [99], possibly as part complex mechanisms leading to a reduction of the risk of $\mathrm{CV}$ events associated with this intervention in patients with chronic inflammatory diseases [101].

4.3. Osteopontin $(\mathrm{OPN})$. Osteopontin (OPN) is another biomarker of atherosclerosis, synthesized by osteoclasts, osteoblasts, chondrocytes, and by cells of the immune system $[102,103]$. This protein has pleiotropic functions such as cellular adhesion, migration, angiogenesis, and inflammation $[102,104]$. High OPN levels have been proposed to promote the development of atherosclerotic lesions [105] and atherosclerotic plaque rupture, acting as a chemotactic factor for inflammatory cells and leading thus to plaque rupture [106].

In a previous study performed by Choi et al., OPN levels were increased in AS when compared to healthy controls [102]. However, we did not observe significant differences in the levels of OPN between AS patients undergoing antiTNF- $\alpha$ therapy and controls [107]. These different results may be due to the long-term treatment with anti-TNF- $\alpha$ therapy that our AS patients had received at the time of the study. It is possible that prolonged anti-TNF- $\alpha$ blockade may lead to reduction of the inflammatory burden and, therefore, to a reduction of OPN concentrations. In this regard, OPN levels observed in our cohort of AS patients and in the healthy matched controls were very similar to those observed in the control group reported by Choi et al. [102].

Interestingly, in our series of AS patients we found a positive correlation between serum levels of OPN and Angpt2 [107]. Since, as described in the previous section, Angpt-2 is a marker of endothelial cell activation involved in angiogenesis, making the endothelium responsive to inflammatory cytokines [97], while OPN is also involved in the development of atherosclerotic disease, this result reinforces the idea of the potential use of OPN and Angpt-2 as biomarkers to predict CV risk in patients with AS.

As previously mentioned, a single infusion of the antiTNF- $\alpha$ monoclonal antibody infliximab led to a significant reduction in Angpt-2 serum levels in our series of AS patients [99]. Likewise, a single infusion of infliximab also triggered a decrease in OPN serum levels in our cohort of AS patients [107]. This is in accordance with the proinflammatory role proposed for these biomarkers of endothelial cell activation and atherosclerosis and the beneficial effect against the development of CV disease mediated by the use of anti-TNF$\alpha$ therapy.

4.4. Gelsolin (GSN). Gelsolin (GSN) is an anti-inflammatory protein mainly secreted by muscle cells, that is, involved in cytoskeleton reorganization [108]. GSN acts by binding to actin filaments (and probably to other extracellular matrix components) and thus prevents the activation of downstream inflammatory pathways by these filaments [109]. Reduced GSN levels have been reported in situations of acute injury or inflammation [109-111]. In this regard, it was proposed that in inflammatory diseases such as RA, circulating GSN may be potentially locally consumed by the interaction with macromolecules such as actin, fibrin, and fibronectin at the joints or other affected organs, leading thus to a reduction of the levels of GSN [109]. In keeping with these findings we observed lower levels of GSN in AS patients undergoing anti-TNF- $\alpha$ therapy when compared to healthy controls [112]. These data support the potential function of GSN as an antiinflammatory molecule.

As observed for other biomarkers and adipokines, a single infusion of anti-TNF- $\alpha$ infliximab did not produce any significant change on GSN serum levels in our AS patients [112]. As pointed out before, a possible explanation for this steady level of GSN might be the low disease activity of our patients, since they had been receiving infliximab for a long period of time.

4.5. Osteoprotegerin $(\mathrm{OPG})$. Osteoprotegerin (OPG) is a member of the TNF receptor superfamily, that is, implicated both in osteoporosis and in the atherosclerotic process. OPG acts as a decoy receptor for the receptor activator of nuclear factor- $\kappa \mathrm{B}$ ligand (RANKL), inhibiting binding of RANKL to its receptor, RANK [113, 114]. OPG also acts as a soluble neutralizing receptor of TNF-related apoptosisinducing ligand (TRAIL), an anti-inflammatory molecule with antiatherosclerotic properties [115-117]. Furthermore, it has been reported that OPG can upregulate the production of endothelial adhesion molecules [118].

OPG has previously been associated with increased risk of atherosclerotic disease in the general population [119]. Interestingly, a recent study of our group on patients with RA undergoing infliximab therapy disclosed that OPG concentrations were associated with biomarkers of endothelial activation (intercellular adhesion molecule-1), carotid intima-media wall thickness, and carotid plaques [120]. In keeping with these results, in our series of AS patients undergoing infliximab therapy we have disclosed an independent correlation with ADMA, another biomarker of endothelial cell activation [121]. This further supports the role of OPG as a valuable CV disease risk biomarker in chronic inflammatory rheumatic diseases such as AS.

Although a significant reduction of OPG levels upon infusion of this biologic agent was recently reported in longstanding RA patients with severe disease undergoing antiTNF- $\alpha$ therapy [120], a single administration of anti-TNF$\alpha$ infliximab did not lead to any significant reduction of 
OPG levels in our series of AS patients [121]. The low disease activity and low inflammatory burden observed at the time of the study in our series of AS patients could probably explain the lack of significant reduction of OPG levels following administration of anti-TNF- $\alpha$.

\section{Therapeutic Potential Applications of These Biomarkers and Adipokines}

Constantly, a progressively increasing list of new potential biomarkers and adipokines comes out, being subject to evaluation for their involvement in $\mathrm{CV}$ disease or MeS. However, as wisely suggested by other authors, these molecules exert such complex physiological effects that their use as potential therapeutic molecules must be carefully planned to obtain the adequate result and to avoid unknown side effects $[5,40]$.

Another point of potential interest is the possible use of these molecules as biomarkers of $\mathrm{CV}$ disease and predictors of increased risk for $\mathrm{CV}$ events or $\mathrm{MeS}$ in patients with diseases like AS that are associated with increased risk of $\mathrm{CV}$ death. However, up to now the routine use of these biomarkers in the daily clinical practice is still far from being well established. It is applicable not only to individuals with chronic inflammatory rheumatic diseases like AS or RA but also to the general population.

\section{Conclusions}

The assessment of adipokines and biomarkers of endothelial cell activation and MeS may be of potential interest for the improvement of the stratification of the $\mathrm{CV}$ risk of patients with AS. However, further studies are still needed to fully elucidate the clinical implication of these molecules in the mechanisms leading to accelerated atherosclerosis in AS and the benefits of the assessment of these molecules in the daily clinical practice.

\section{Conflict of Interests}

The authors declare that there is no conflict of interests regarding the publication of this paper.

\section{Acknowledgments}

The authors thank Mrs. Susana Escandon and Isabel CastroFernandez, nurses from the Rheumatology Outpatient Clinic, and Ms. Pilar Ruiz, a nurse from the Hematology Division, and the members of the Biochemistry Department from Hospital Lucus Augusti, Lugo (Spain) for their valuable help to undertake this study. This study was supported by European Union FEDER funds and "Fondo de Investigación Sanitaria" (Grants PI06/0024, PS09/00748, and PI12/00060) (Spain). This work was also partially supported by RETICS Programs, RD08/0075 (RIER) and RD12/0009/0013, from "Instituto de Salud Carlos III" (ISCIII) (Spain). FG is supported by funds from the RETICS Program (RIER). RLM is a recipient of a Sara Borrell postdoctoral fellowship from the Instituto Carlos III de Salud at the Spanish Ministry of Health (Spain).

\section{References}

[1] A. El Maghraoui, "Extra-articular manifestations of ankylosing spondylitis: prevalence, characteristics and therapeutic implications," European Journal of Internal Medicine, vol. 22, no. 6, pp. 554-560, 2011.

[2] J. Braun, R. van den Berg, X. Baraliakos et al., "2010 update of the ASAS/EULAR recommendations for the management of ankylosing spondylitis," Annals of the Rheumatic Diseases, vol. 70, no. 6, pp. 896-904, 2011.

[3] C. Gonzalez-Juanatey, T. R. Vazquez-Rodriguez, J. A. MirandaFilloy et al., "The high prevalence of subclinical atherosclerosis in patients with ankylosing spondylitis without clinically evident cardiovascular disease," Medicine, vol. 88, no. 6, pp. 358$365,2009$.

[4] S. Mathieu, P. Motreff, and M. Soubrier, "Spondyloarthropathies: an independent cardiovascular risk factor?" Joint Bone Spine, vol. 77, no. 6, pp. 542-545, 2010.

[5] Y. Deng and P. E. Scherer, "Adipokines as novel biomarkers and regulators of the metabolic syndrome," Annals of the New York Academy of Sciences, vol. 1212, pp. E1-E19, 2010.

[6] C. Procaccini, V. de Rosa, M. Galgani et al., "Role of adipokines signaling in the modulation of T cells function," Frontiers in Immunology, vol. 4, Article ID 332, 2013.

[7] S. E. Shoelson, J. Lee, and A. B. Goldfine, "Inflammation and insulin resistance," Journal of Clinical Investigation, vol. 116, no. 7, pp. 1793-1801, 2006.

[8] J. A. Kim, M. Montagnani, K. K. Kwang, and M. J. Quon, "Reciprocal relationships between insulin resistance and endothelial dysfunction: molecular and pathophysiological mechanisms," Circulation, vol. 113, no. 15, pp. 1888-1904, 2006.

[9] E. Cersosimo and R. A. DeFronzo, "Insulin resistance and endothelial dysfunction: the road map to cardiovascular diseases," Diabetes/Metabolism Research and Reviews, vol. 22, no. 6, pp. 423-436, 2006.

[10] I. Sari, T. Okan, S. Akar et al., "Impaired endothelial function in patients with ankylosing spondylitis," Rheumatology, vol. 45, no. 3, pp. 283-286, 2006.

[11] S. D’Angelo, C. Palazzi, F. Cantini et al., "Etanercept in spondyloarthropathies. Part II. Safety and pharmacoeconomic issues," Clinical and Experimental Rheumatology, vol. 29, no. 5, pp. 865870, 2011

[12] C. Palazzi, S. D’Angelo, F. Cantini et al., "Etanercept in spondyloarthropathies. Part I. Current evidence of efficacy," Clinical and Experimental Rheumatology, vol. 29, no. 5, pp. 858-864, 2011.

[13] F. Heldmann, J. Brandt, I. E. van der Horst-Bruinsma et al., "The European ankylosing spondylitis infliximab cohort (EASIC): a European multicentre study of long term outcomes in patients with ankylosing spondylitis treated with infliximab," Clinical and experimental rheumatology, vol. 29, no. 4, pp. 672-680, 2011.

[14] J. S. Smolen and P. Emery, "Infliximab: 12 years of experience," Arthritis Research and Therapy, vol. 13, no. 1, article S2, 2011.

[15] A. Syngle, K. Vohra, A. Sharma, and L. Kaur, "Endothelial dysfunction in ankylosing spondylitis improves after tumor necrosis factor- $\alpha$ blockade," Clinical Rheumatology, vol. 29, no. 7, pp. 763-770, 2010.

[16] J. A. Miranda-Filloy, J. Llorca, B. Carnero-López, C. GonzálezJuanatey, R. Blanco, and M. A. González-Gay, “TNF- $\alpha$ antagonist therapy improves insulin sensitivity in non-diabetic ankylosing spondylitis patients," Clinical Experimental and Rheumatology, vol. 30, no. 6, pp. 850-855, 2012. 
[17] M. A. Gonzalez-Gay, J. M. de Matias, C. Gonzalez-Juanatey et al., "Anti-tumor necrosis factor- $\alpha$ blockade improves insulin resistance in patients with rheumatoid arthritis," Clinical and Experimental Rheumatology, vol. 24, no. 1, pp. 83-86, 2006.

[18] M. A. Gonzalez-Gay, C. Gonzalez-Juanatey, T. R. VazquezRodriguez, J. A. Miranda-Filloy, and J. Llorca, "Insulin resistance in rheumatoid arthritis: the impact of the anti-TNF- $\alpha$ therapy," Annals of the New York Academy of Sciences, vol. 1193, pp. 153-159, 2010.

[19] D. Baatar, K. Patel, and D. D. Taub, "The effects of ghrelin on inflammation and the immune system," Molecular and Cellular Endocrinology, vol. 340, no. 1, pp. 44-58, 2011.

[20] V. D. Dixit, E. M. Schaffer, R. S. Pyle et al., "Ghrelin inhibits leptin- and activation-induced proinflammatory cytokine expression by human monocytes and T cells," Journal of Clinical Investigation, vol. 114, no. 1, pp. 57-66, 2004.

[21] M. Tschöp, C. Weyer, P. A. Tataranni, V. Devanarayan, E. Ravussin, and M. L. Heiman, "Circulating ghrelin levels are decreased in human obesity," Diabetes, vol. 50, no. 4, pp. 707709, 2001.

[22] F. Genre, R. López-Mejias, J. A. Miranda-Filloy et al., "Correlation between insulin resistance and serum ghrelin in nondiabetic ankylosing spondylitis patients undergoing anti-TNF$\alpha$ therapy," Clinical and Experimental Rheumatology, vol. 31, no. 6, pp. 913-918, 2013.

[23] S. M. Pöykkö, E. Kellokoski, S. Hörkkö, H. Kauma, Y. A. Kesäniemi, and O. Ukkola, "Low plasma ghrelin is associated with insulin resistance, hypertension, and the prevalence of type 2 diabetes," Diabetes, vol. 52, no. 10, pp. 2546-2553, 2003.

[24] M. A. Lazar, "Resistin- and obesity-associated metabolic diseases," Hormone and Metabolic Research, vol. 39, no. 10, pp. 710716, 2007.

[25] É. Toussirot, G. Streit, N. U. Nguyen et al., "Adipose tissue, serum adipokines, and ghrelin in patients with ankylosing spondylitis," Metabolism, vol. 56, no. 10, pp. 1383-1389, 2007.

[26] M. Otero, R. Nogueiras, F. Lago, C. Dieguez, J. J. Gomez-Reino, and O. Gualillo, "Chronic inflammation modulates ghrelin levels in humans and rats," Rheumatology, vol. 43, no. 3, pp. 306310, 2004.

[27] M. A. Gonzalez-Gay, M. T. Garcia-Unzueta, A. Berja et al., "Anti-tumour necrosis factor $\alpha$ therapy modulates ghrelin in patients with severe rheumatoid arthritis," Annals of the Rheumatic Diseases, vol. 67, no. 11, pp. 1644-1646, 2008.

[28] H. Kwon and J. E. Pessin, "Adipokines mediate inflammation and insulin resistance," Frontiers in Endocrinology, vol. 4, article 71, 2013.

[29] T. E. Graham, Q. Yang, M. Blüher et al., "Retinol-binding protein 4 and insulin resistance in lean, obese, and diabetic subjects," The New England Journal of Medicine, vol. 354, no. 24, pp. 2552-2563, 2006.

[30] S. Gavi, L. M. Stuart, P. Kelly et al., "Retinol-binding protein 4 is associated with insulin resistance and body fat distribution in nonobese subjects without type 2 diabetes," Journal of Clinical Endocrinology and Metabolism, vol. 92, no. 5, pp. 1886-1890, 2007.

[31] G. A. Christou, A. D. Tselepis, and D. N. Kiortsis, "The metabolic role of retinol binding protein 4: an update," Hormone and Metabolic Research, vol. 44, no. 1, pp. 6-14, 2012.

[32] F. Genre, R. López-Mejías, J. A. Miranda-Filloy et al., "Antitumour necrosis factor- $\alpha$ treatment reduces retinol-binding protein 4 serum levels in non-diabetic ankylosing spondylitis patients," Annals of the Rheumatic Diseases, 2013.
[33] F. D. O'Shea, F. W. L. Tsui, B. Chiu, H. W. Tsui, M. Yazdanpanah, and R. D. Inman, "Retinol (vitamin A) and retinol-binding protein levels are decreased in ankylosing spondylitis: clinical and genetic analysis," Journal of Rheumatology, vol. 34, no. 12, pp. 2457-2459, 2007.

[34] D. K. Oh, T. Ciaraldi, and R. R. Henry, "Adiponectin in health and disease," Diabetes, Obesity and Metabolism, vol. 9, no. 3, pp. 282-289, 2007.

[35] M. Otero, R. Logo, R. Gomez et al., "Changes in plasma levels of fat-derived hormones adiponectin, leptin, resistin and visfatin in patients with rheumatoid arthritis," Annals of the Rheumatic Diseases, vol. 65, no. 9, pp. 1198-1201, 2006.

[36] C. S. Derdemezis, T. D. Filippatos, P. V. Voulgari, A. D. Tselepis, A. A. Drosos, and D. N. Kiortsis, "Leptin and adiponectin levels in patients with ankylosing spondylitis. The effect of infliximab treatment," Clinical and Experimental Rheumatology, vol. 28, no. 6, pp. 880-883, 2010.

[37] J. A. Miranda-Filloy, R. López-Mejias, F. Genre et al., "Adiponectin and resistin serum levels in non-diabetic ankylosing spondylitis patients undergoing TNF- $\alpha$ antagonist therapy," Clinical and Experimental Rheumatology, vol. 31, no. 3, pp. 365-371, 2013.

[38] T. Yamauchi, J. Kamon, Y. Minokoshi et al., "Adiponectin stimulates glucose utilization and fatty-acid oxidation by activating AMP-activated protein kinase," Nature Medicine, vol. 8, no. 11, pp. 1288-1295, 2002.

[39] M. A. Gonzalez-Gay, J. Llorca, M. T. Garcia-Unzueta et al., "High-grade inflammation, circulating adiponectin concentrations and cardiovascular risk factors in severe rheumatoid arthritis," Clinical and Experimental Rheumatology, vol. 26, no. 4, pp. 596-603, 2008.

[40] E. Neumann, K. W. Frommer, M. Vasile, and U. Müller-Ladner, "Adipocytokines as driving forces in rheumatoid arthritis and related inflammatory diseases?" Arthritis and Rheumatism, vol. 63, no. 5, pp. 1159-1169, 2011.

[41] H. B. Hartman, X. Hu, K. X. Tyler, C. K. Dalal, and M. A. Lazar, "Mechanisms regulating adipocyte expression of resistin," Journal of Biological Chemistry, vol. 277, no. 22, pp. 19754-19761, 2002.

[42] K. Migita, Y. Maeda, T. Miyashita et al., "The serum levels of resistin in rheumatoid arthritis patients," Clinical and Experimental Rheumatology, vol. 24, no. 6, pp. 698-701, 2006.

[43] L. Šenolt, D. Housa, Z. Vernerová et al., "Resistin in rheumatoid arthritis synovial tissue, synovial fluid and serum," Annals of the Rheumatic Diseases, vol. 66, no. 4, pp. 458-463, 2007.

[44] M. A. Gonzalez-Gay, M. T. Garcia-Unzueta, C. GonzalezJuanatey et al., "Anti-TNF- $\alpha$ therapy modulates resistin in patients with rheumatoid arthritis," Clinical and Experimental Rheumatology, vol. 26, no. 2, pp. 311-316, 2008.

[45] A. Schäffler, A. Ehling, E. Neumann et al., "Adipocytokines in synovial fluid," Journal of the American Medical Association, vol. 290, no. 13, pp. 1709-1710, 2003.

[46] H. Kocabas, V. Kocabas, S. Buyukbas, M. A. Melikoglu, I. Sezer, and B. Butun, "The serum levels of resistin in ankylosing spondylitis patients: a pilot study," Rheumatology International, vol. 32, no. 3, pp. 699-702, 2012.

[47] G. Fantuzzi and R. Faggioni, "Leptin in the regulation of immunity, inflammation, and hematopoiesis," Journal of Leukocyte Biology, vol. 68, no. 4, pp. 437-446, 2000.

[48] H. Tilg and A. R. Moschen, "Adipocytokines: mediators linking adipose tissue, inflammation and immunity," Nature Reviews Immunology, vol. 6, no. 10, pp. 772-783, 2006. 
[49] B. Targońska-Stẹpniak, M. Dryglewska, and M. Majdan, "Adiponectin and leptin serum concentrations in patients with rheumatoid arthritis," Rheumatology International, vol. 30, no. 6, pp. 731-737, 2010.

[50] T. Gainsford, T. A. Willson, D. Metcalf et al., "Leptin can induce proliferation, differentiation, and functional activation of hemopoietic cells," Proceedings of the National Academy of Sciences of the United States of America, vol. 93, no. 25, pp. 14564-14568, 1996.

[51] G. M. Lord, G. Matarese, J. K. Howard, R. J. Baker, S. R. Bloom, and R. I. Lechler, "Leptin modulates the T-cell immune response and reverses starvation- induced immunosuppression," Nature, vol. 394, no. 6696, pp. 897-901, 1998.

[52] M. Otero, R. Lago, R. Gómez, F. Lago, J. J. Gómez-Reino, and O. Gualillo, "Leptin: a metabolic hormone that functions like a proinflammatory adipokine," Drug News and Perspectives, vol. 19, no. 1, pp. 21-26, 2006.

[53] T. Yoshino, N. Kusunoki, N. Tanaka et al., "Elevated serum levels of resistin, leptin, and adiponectin are associated with Creactive protein and also other clinical conditions in rheumatoid arthritis," Internal Medicine, vol. 50, no. 4, pp. 269-275, 2011.

[54] M. C. Park, S. J. Chung, Y. B. Park, and S. K. Lee, "Proinflammatory effect of leptin on peripheral blood mononuclear cells of patients with ankylosing spondylitis," Joint Bone Spine, vol. 76, no. 2, pp. 170-175, 2009.

[55] I. Sari, T. Demir, L. D. Kozaci et al., "Body composition, insulin, and leptin levels in patients with ankylosing spondylitis," Clinical Rheumatology, vol. 26, no. 9, pp. 1427-1432, 2007.

[56] J. A. Miranda-Filloy, R. López-Mejias, F. Genre et al., "Leptin and visfatin serum levels in non-diabetic ankylosing spondylitis patients undergoing TNF- $\alpha$ antagonist therapy," Clinical and Experimental Rheumatology, vol. 31, no. 4, pp. 538-545, 2013.

[57] M. A. Gonzalez-Gay, M. T. Garcia-Unzueta, A. Berja et al., "Anti-TNF- $\alpha$ therapy does not modulate leptin in patients with severe rheumatoid arthritis," Clinical and Experimental Rheumatology, vol. 27, no. 2, pp. 222-228, 2009.

[58] B. J. Nicklas, "Gender differences in the response of plasma leptin concentrations to weight loss in obese older individuals," Obesity Research, vol. 5, no. 1, pp. 62-68, 1997.

[59] C. Popa, M. G. Netea, J. De Graaf et al., "Circulating leptin and adiponectin concentrations during tumor necrosis factor blockade in patients with active rheumatoid arthritis," Journal of Rheumatology, vol. 36, no. 4, pp. 724-730, 2009.

[60] T. Luk, Z. Malam, and J. C. Marshall, "Pre-B cell colonyenhancing factor (PBEF)/visfatin: a novel mediator of innate immunity," Journal of Leukocyte Biology, vol. 83, no. 4, pp. 804816, 2008.

[61] A. Fukuhara, M. Matsuda, M. Nishizawa et al., "Visfatin: a protein secreted by visceral fat that Mimics the effects of insulin," Science, vol. 307, no. 5708, pp. 426-430, 2005.

[62] J. Berndt, N. Klöting, S. Kralisch et al., "Plasma visfatin concentrations and fat depot-specific mRNA expression in humans," Diabetes, vol. 54, no. 10, pp. 2911-2916, 2005.

[63] F. Brentano, O. Schorr, C. Ospelt et al., "Pre-B cell colonyenhancing factor/visfatin, a new marker of inflammation in rheumatoid arthritis with proinflammatory and matrixdegrading activities," Arthritis and Rheumatism, vol. 56, no. 9, pp. 2829-2839, 2007.

[64] A. R. Moschen, A. Kaser, B. Enrich et al., "Visfatin, an adipocytokine with proinflammatory and immunomodulating properties," Journal of Immunology, vol. 178, no. 3, pp. 1748-1758, 2007.
[65] H. Hulejová, A. Levitová, M. Kuklová et al., "No effect of physiotherapy on the serum levels of adipocytokines in patients with ankylosing spondylitis," Clinical Rheumatology, vol. 31, no. 1, pp. 67-71, 2012.

[66] M. A. Gonzalez-Gay, T. R. Vazquez-Rodriguez, M. T. GarciaUnzueta et al., "Visfatin is not associated with inflammation or metabolic syndrome in patients with severe rheumatoid arthritis undergoing anti-TNF- $\alpha$ therapy," Clinical and Experimental Rheumatology, vol. 28, no. 1, pp. 56-62, 2010.

[67] I. Kowalska, M. Straczkowski, A. Nikolajuk et al., "Serum visfatin in relation to insulin resistance and markers of hyperandrogenism in lean and obese women with polycystic ovary syndrome," Human Reproduction, vol. 22, no. 7, pp. 1824-1829, 2007.

[68] H. Kidoya and N. Takakura, "Biology of the apelin-APJ axis in vascular formation," Journal of Biochemistry, vol. 152, no. 2, pp. 125-131, 2012.

[69] A. G. Japp, N. L. Cruden, D. A. B. Amer et al., "Vascular effects of apelin in vivo in man," Journal of the American College of Cardiology, vol. 52, no. 11, pp. 908-913, 2008.

[70] J. Boucher, B. Masri, D. Daviaud et al., "Apelin, a newly identified adipokine up-regulated by insulin and obesity," Endocrinology, vol. 146, no. 4, pp. 1764-1771, 2005.

[71] I. Tasci, T. Dogru, I. Naharci et al., "Plasma apelin is lower in patients with elevated LDL-cholesterol," Experimental and Clinical Endocrinology and Diabetes, vol. 115, no. 7, pp. 428-432, 2007.

[72] J. Malyszko, J. S. Malyszko, K. Pawlak, and M. Mysliwiec, "Visfatin and apelin, new adipocytokines, and their relation to endothelial function in patients with chronic renal failure," Advances in Medical Sciences, vol. 53, no. 1, pp. 32-36, 2008.

[73] Z. Li, Y. Bai, and J. Hu, "Reduced apelin levels in stable angina," Internal Medicine, vol. 47, no. 22, pp. 1951-1955, 2008.

[74] L. Li, G. Yang, Q. Li et al., "Changes and relations of circulating visfatin, apelin, and resistin levels in normal, impaired glucose tolerance, and type 2 diabetic subjects," Experimental and Clinical Endocrinology and Diabetes, vol. 114, no. 10, pp. 544548, 2006.

[75] G. Erdem, T. Dogru, I. Tasci, A. Sonmez, and S. Tapan, "Low plasma apelin levels in newly diagnosed type 2 diabetes mellitus," Experimental and Clinical Endocrinology and Diabetes, vol. 116 , no. 5, pp. 289-292, 2008.

[76] M. di Franco, F. R. Spinelli, A. Metere et al., "Serum levels of asymmetric dimethylarginine and apelin as potential markers of vascular endothelial dysfunction in early rheumatoid arthritis," Mediators of Inflammation, vol. 2012, Article ID 347268, 7 pages, 2012.

[77] F. Genre, J. A. Miranda-Filloy, R. López-Mejias et al., "Apelin serum levels in non-diabetic ankylosing spondylitis patients undergoing TNF- $\alpha$ antagonist therapy," Clinical and Experimental Rheumatology, vol. 31, no. 4, pp. 532-537, 2013.

[78] I. Ferraz-Amaro, M. Arce-Franco, J. Muiz et al., "Systemic blockade of TNF- $\alpha$ does not improve insulin resistance in humans," Hormone and Metabolic Research, vol. 43, no. 11, pp. 801-808, 2011.

[79] J. E. Deanfield, J. P. Halcox, and T. J. Rabelink, "Endothelial function and dysfunction: testing and clinical relevance," Circulation, vol. 115, no. 10, pp. 1285-1295, 2007.

[80] D. Tousoulis, A.-M. Kampoli, C. T. N. Papageorgiou, and C. Stefanadis, "The role of nitric oxide on endothelial function," Current Vascular Pharmacology, vol. 10, no. 1, pp. 4-18, 2012. 
[81] P. Vallance and N. Chan, "Endothelial function and nitric oxide: clinical relevance," Heart, vol. 85, no. 3, pp. 342-350, 2001.

[82] L. Sibal, S. C. Agarwal, P. D. Home, and R. H. Boger, "The role of asymmetric dimethylarginine (ADMA) in endothelial dysfunction and cardiovascular disease," Current Cardiology Reviews, vol. 6, no. 2, pp. 82-90, 2010.

[83] R. H. Böger, "The emerging role of asymmetric dimethylarginine as a novel cardiovascular risk factor," Cardiovascular Research, vol. 59, no. 4, pp. 824-833, 2003.

[84] A. Surdacki, M. Nowicki, J. Sandmann et al., "Reduced urinary excretion of nitric oxide metabolites and increased plasma levels of asymmetric dimethylarginine in men with essential hypertension," Journal of Cardiovascular Pharmacology, vol. 33, no. 4, pp. 652-658, 1999.

[85] P. Lundman, M. J. Eriksson, M. Stühlinger, J. P. Cooke, A. Hamsten, and P. Tornvall, "Mild-to-moderate hypertriglyceridemia in young men is associated with endothelial dysfunction and increased plasma concentrations of asymmetric dimethylarginine," Journal of the American College of Cardiology, vol. 38, no. 1, pp. 111-116, 2001.

[86] R. H. Böger, S. M. Bode-Böger, A. Szuba et al., "Asymmetric dimethylarginine (ADMA): a novel risk factor for endothelial dysfunction: its role in hypercholesterolemia," Circulation, vol. 98, no. 18, pp. 1842-1847, 1998.

[87] F. Abbasi, T. Asagmi, J. P. Cooke et al., "Plasma concentrations of asymmetric dimethylarginine are increased in patients with type 2 diabetes mellitus," The American Journal of Cardiology, vol. 88, no. 10, pp. 1201-1203, 2001.

[88] M. C. Stühlinger, F. Abbasi, J. W. Chu et al., "Relationship between insulin resistance and an endogenous nitric oxide synthase inhibitor," The Journal of the American Medical Association, vol. 287, no. 11, pp. 1420-1426, 2002.

[89] I. Sari, L. Kebapcilar, A. Alacacioglu et al., "Increased levels of asymmetric dimethylarginine (ADMA) in patients with ankylosing spondylitis," Internal Medicine, vol. 48, no. 16, pp. 1363-1368, 2009.

[90] Á. Kemény-Beke, R. Gesztelyi, N. Bodnár et al., "Increased production of asymmetric dimethylarginine (ADMA) in ankylosing spondylitis: association with other clinical and laboratory parameters," Joint Bone Spine, vol. 78, no. 2, pp. 184-187, 2011.

[91] G. L. Erre, P. Sanna, A. Zinellu et al., "Plasma asymmetric dimethylarginine (ADMA) levels and atherosclerotic disease in ankylosing spondylitis: a cross-sectional study," Clinical Rheumatology, vol. 30, no. 1, pp. 21-27, 2011.

[92] F. Genre, R. López-Mejías, J. A. Miranda-Filloy et al., "Asymmetric dimethylarginine serum levels in non-diabetic ankylosing spondylitis patients undergoing TNF- $\alpha$ antagonist therapy," Clinical and Experimental Rheumatology, vol. 31, no. 5, pp. 749755, 2013.

[93] C. Popa, F. H. van den Hoogen, T. R. Radstake et al., "Modulation of lipoprotein plasma concentrations during long-term anti-TNF therapy in patients with active rheumatoid arthritis," Annals of the Rheumatic Diseases, vol. 66, no. 11, pp. 1503-1507, 2007.

[94] C. D. Popa, E. Arts, J. Fransen, and P. L. van Riel, "Atherogenic index and high-density lipoprotein cholesterol as cardiovascular risk determinants in rheumatoid arthritis: the impact of therapy with biologicals," Mediators of Inflammation, vol. 2012, Article ID 785946, 9 pages, 2012.

[95] K. Angel, S. A. Provan, P. Mowinckel, I. Seljeflot, T. K. Kvien, and D. Atar, "The L-arginine/asymmetric dimethylarginine ratio is improved by anti-tumor necrosis factor- $\alpha$ therapy in inflammatory arthropathies. Associations with aortic stiffness," Atherosclerosis, vol. 225, no. 1, pp. 160-165, 2012.

[96] M. Korkosz, J. Gąsowski, A. Surdacki et al., "Disparate effects of anti-TNF- $\alpha$ therapies on measures of disease activity and mediators of endothelial damage in ankylosing spondylitis," Pharmacological Reports, vol. 65, no. 4, pp. 891-897, 2013.

[97] J. Westra, L. de Groot, S. L. Plaxton et al., "Angiopoietin-2 is highly correlated with inflammation and disease activity in recent-onset rheumatoid arthritis and could be predictive for cardiovascular disease," Rheumatology, vol. 50, no. 4, pp. 665673, 2011

[98] R. López-Mejías, A. Corrales, F. Genre et al., "Angiopoietin-2 serum levels correlate with severity, early onset and cardiovascular disease in patients with rheumatoid arthritis," Clinical and Experimental Rheumatology, vol. 31, no. 5, pp. 761-766, 2013.

[99] F. Genre, J. A. Miranda-Filloy, R. López-Mejias et al., "Antitumour necrosis factor- $\alpha$ therapy modulates angiopoietin-2 serum levels in non-diabetic ankylosing spondylitis patients," Annals of the Rheumatic Diseases, vol. 72, no. 7, pp. 1265-1267, 2013.

[100] S. Krausz, S. Garcia, C. A. Ambarus et al., "Angiopoietin-2 promotes inflammatory activation of human macrophages and is essential for murine experimental arthritis," Annals of the Rheumatic Diseases, vol. 71, no. 8, pp. 1402-1410, 2012.

[101] J. D. Greenberg, J. M. Kremer, J. R. Curtis et al., "Tumour necrosis factor antagonist use and associated risk reduction of cardiovascular events among patients with rheumatoid arthritis," Annals of the Rheumatic Diseases, vol. 70, no. 4, pp. 576-582, 2011.

[102] S. T. Choi, J. H. Kim, E. J. Kang et al., "Osteopontin might be involved in bone remodelling rather than in inflammation in ankylosing spondylitis," Rheumatology, vol. 47, no. 12, pp. 17751779, 2008.

[103] T. A. Briggs, "Osteopontin-a biomarker for organ damage in paediatric lupus?” Arthritis Research \& Therapy, vol. 15, no. 2, article 110, 2013.

[104] D. N. Haylock and S. K. Nilsson, "Osteopontin: a bridge between bone and blood," British Journal of Haematology, vol. 134, no. 5, pp. 467-474, 2006.

[105] G. Gürsoy, S. Alagöz, Y. Acar, B. Demirbaş, H. Çetiner, and Z. Kiliç, "Osteopontin a new probable marker for atherosclerosis in obese women?" Clinical Reviews and Opinions, vol. 2, no. 3, pp. 35-40, 2010.

[106] J. Golledge, M. McCann, S. Mangan, A. Lam, and M. Karan, "Osteoprotegerin and osteopontin are expressed at high concentrations within symptomatic carotid atherosclerosis," Stroke, vol. 35, no. 7, pp. 1636-1641, 2004.

[107] F. Genre, R. López-Mejias, J. A. Miranda-Filloy et al., "Correlation between two biomarkers of atherosclerosis, osteopontin and angiopoyetin-2, in non-diabetic ankylosing spondylitis patients undergoing TNF- $\alpha$ antagonist therapy," Clinical and Experimental Rheumatology. In press.

[108] D. J. Kwiatkowski, R. Mehl, S. Izumo, B. Nadal-Ginard, and H. L. Yin, "Muscle is the major source of plasma gelsolin," Journal of Biological Chemistry, vol. 263, no. 17, pp. 8239-8243, 1988.

[109] T. M. Osborn, M. Verdrengh, T. P. Stossel, and M. Bokarewa, "Decreased levels of the gelsolin plasma isoform in patients with rheumatoid arthritis," Arthritis Research \& Therapy, vol. 10, no. 5, article R117, 2008.

[110] L. F. Huang, Y. M. Yao, J. F. Li et al., "Reduction of plasma gelsolin levels correlates with development of multiple organ 
dysfunction syndrome and fatal outcome in burn patients," PLoS ONE, vol. 6, no. 11, Article ID e25748, 2011.

[111] V. Aidinis, P. Carninci, M. Armaka et al., "Cytoskeletal rearrangements in synovial fibroblasts as a novel pathophysiological determinant of modeled rheumatoid arthritis," PLoS Genetics, vol. 1, no. 4, article e48, 2005.

[112] F. Genre, R. López-Mejias, J. A. Miranda-Filloy et al., "Gelsolin levels are decreased in ankylosing spondylitis patients undergoing anti-TNF-alpha therapy," Clinical and Experimental Rheumatology. In press.

[113] L. C. Hofbauer and M. Schoppet, "Clinical implications of the osteoprotegerin/RANKL/RANK system for bone and vascular diseases," The Journal of the American Medical Association, vol. 292, no. 4, pp. 490-495, 2004.

[114] A. van Campenhout and J. Golledge, "Osteoprotegerin, vascular calcification and atherosclerosis," Atherosclerosis, vol. 204, no. 2, pp. 321-329, 2009.

[115] P. Secchiero, F. Corallini, A. P. Beltrami et al., "An imbalanced OPG/TRAIL ratio is associated to severe acute myocardial infarction," Atherosclerosis, vol. 210, no. 1, pp. 274-277, 2010.

[116] P. Secchiero, E. Rimondi, M. G. di Lasio et al., "C-reactive protein downregulates TRAIL expression in human peripheral monocytes via an Egr-1-dependent pathway," Clinical Cancer Research, vol. 19, no. 8, pp. 1949-1959, 2013.

[117] B. A. di Bartolo, S. P. Cartland, H. H. Harith, Y. V. Bobryshev, M. Schoppet, and M. M. Kavurma, "TRAIL-deficiency accelerates vascular calcification in atherosclerosis via modulation of RANKL," PLoS ONE, vol. 8, no. 9, Article ID e74211, 2013.

[118] S. H. Mangan, A. V. Campenhout, C. Rush, and J. Golledge, "Osteoprotegerin upregulates endothelial cell adhesion molecule response to tumor necrosis factor- $\alpha$ associated with induction of angiopoietin-2," Cardiovascular Research, vol. 76, no. 3, pp. 494-505, 2007.

[119] S. Kiechl, G. Schett, G. Wenning et al., "Osteoprotegerin is a risk factor for progressive atherosclerosis and cardiovascular disease," Circulation, vol. 109, no. 18, pp. 2175-2180, 2004.

[120] P. H. Dessein, R. L. López-Mejias, C. González-Juanatey et al., "Independent relationship of osteoprotegerin concentrations with endothelial activation and carotid atherosclerosis in patients with severe rheumatoid arthritis," The Journal of Rheumatology, 2014.

[121] F. Genre, R. López-Mejias, J. A. Miranda-Filloy et al., "Osteoprotegerin correlates with disease activity and endothelial activation in non-diabetic ankylosing spondylitis patients undergoing TNF- $\alpha$ antagonist therapy," Clinical and Experimental Rheumatology. In press. 


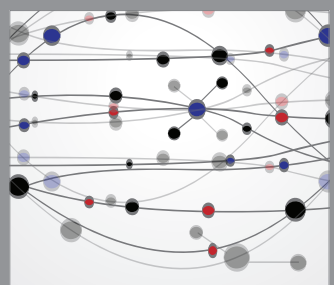

The Scientific World Journal
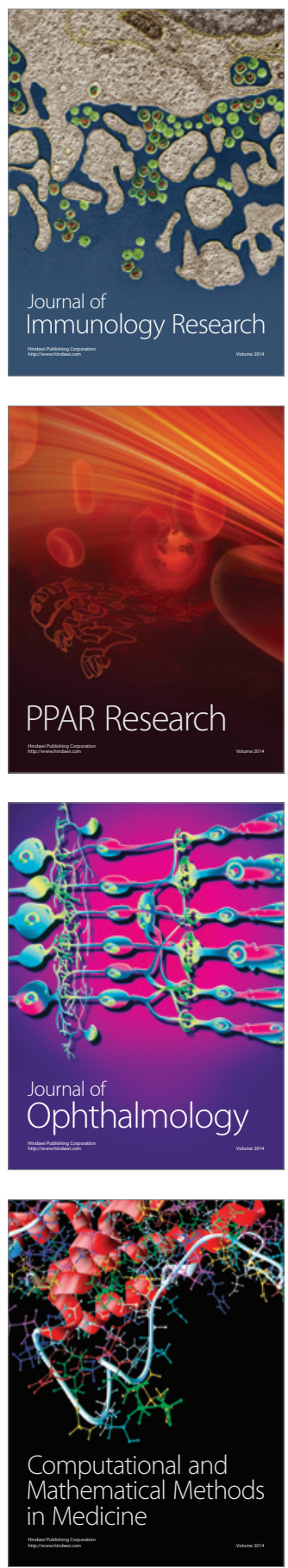

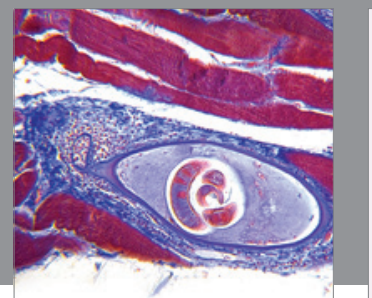

Gastroenterology

Research and Practice
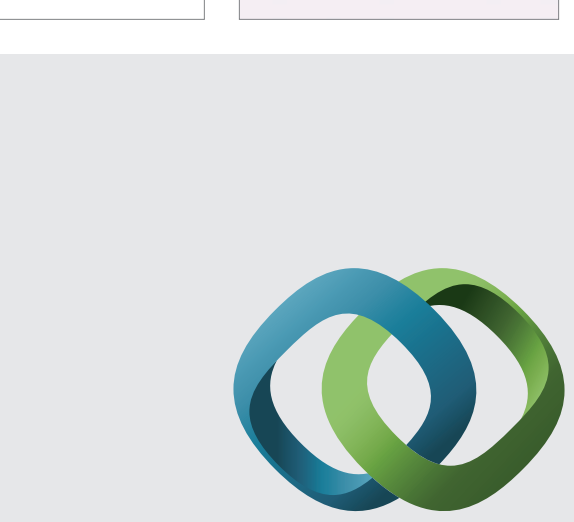

\section{Hindawi}

Submit your manuscripts at

http://www.hindawi.com
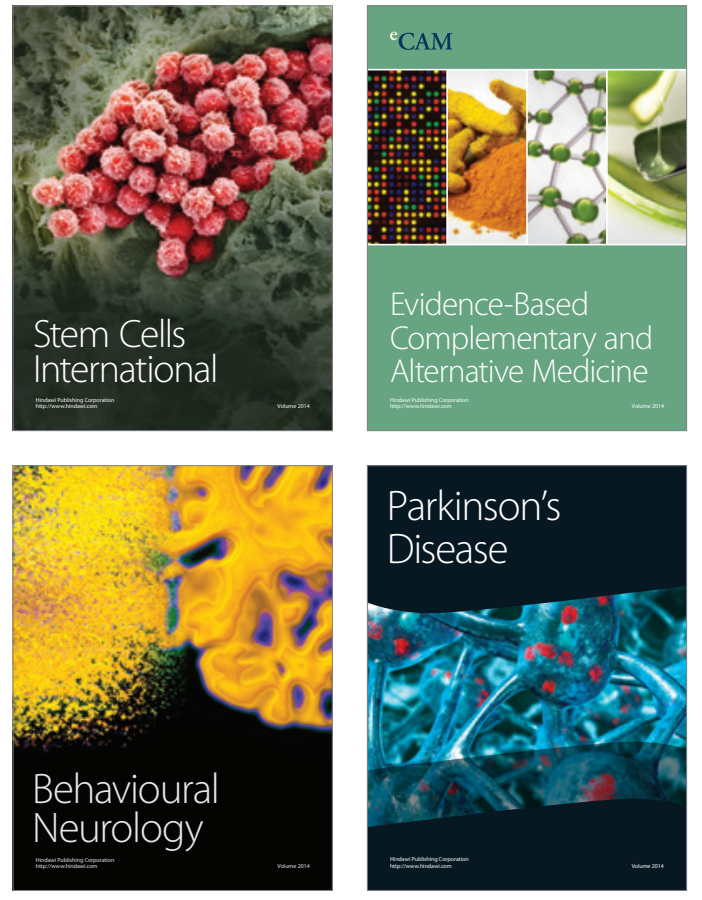
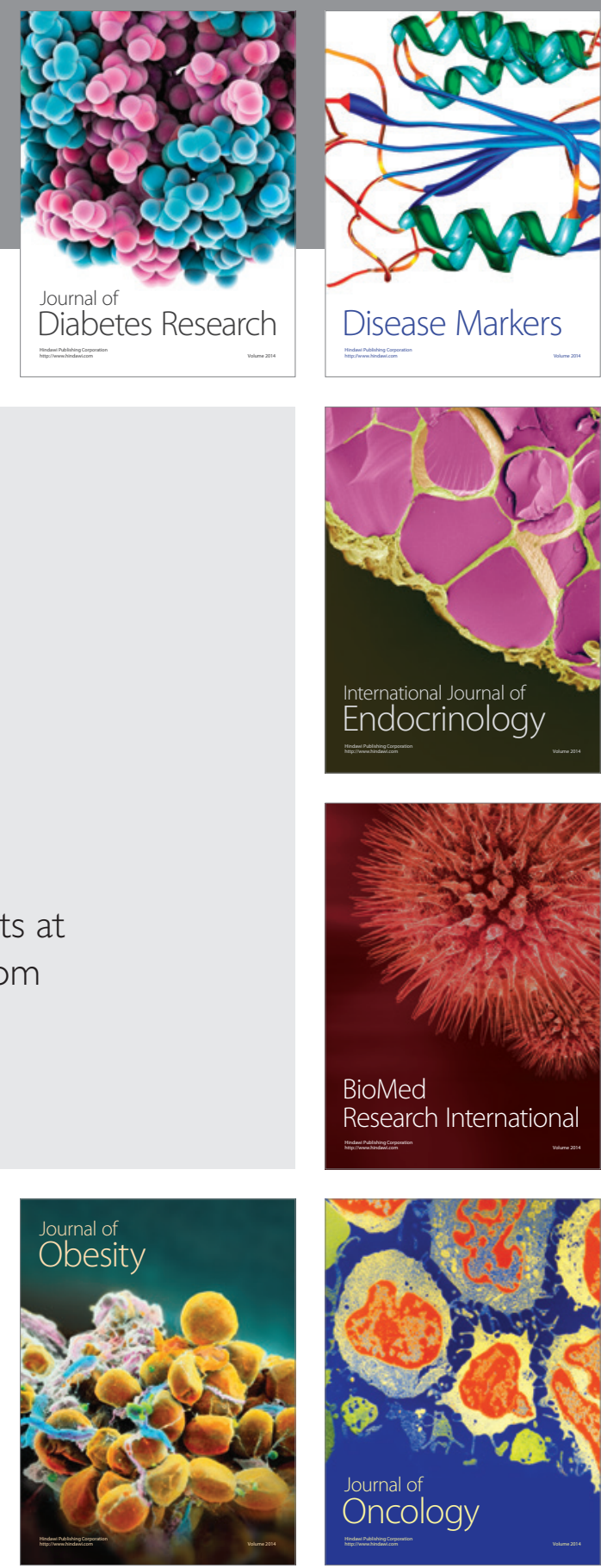

Disease Markers
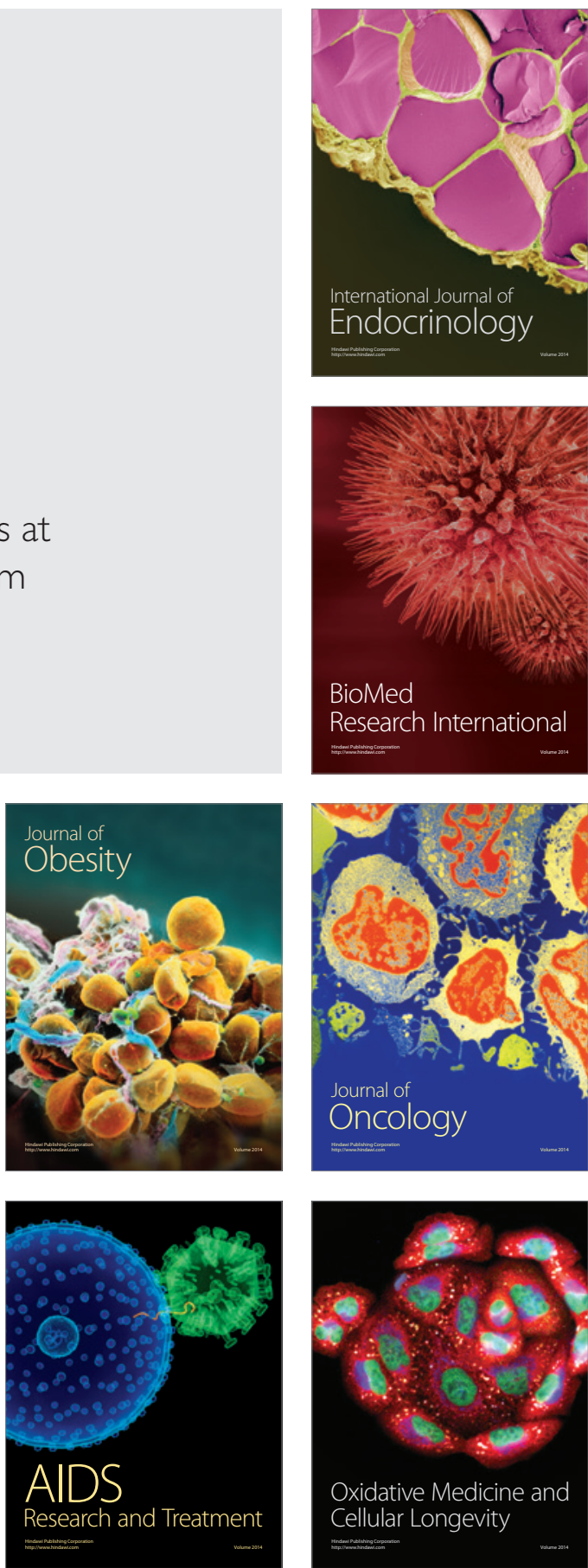